\title{
On the inter-area optimal voltage and reactive power control
}

\author{
Omid Alizadeh Mousavi*, Rachid Cherkaoui \\ Ecole Polytechnique Fédérale de Lausanne (EPFL), 1015 Lausanne, Switzerland
}

\section{A R T I C L E I N F O}

\section{Article history:}

Received 27 November 2012

Received in revised form 1 March 2013

Accepted 13 March 2013

\section{Keywords:}

Multi-Area Power System (MAPS)

Optimal Power Flow (OPF)

Distributed slack bus

Centralized optimization

Decentralized optimization

Non-coordinated operation

\begin{abstract}
A B S T R A C T
Scheduling voltage and reactive power is one of the major problems and concerns of the Transmission System Operators (TSOs) especially after deregulation of power market. Due to locally provision of the reactive power resources, each TSO has developed its own specific method. Hence, the voltage and reactive power coordination in the interconnected system has got less attention. In this respect, current practices of different TSOs in Europe and North America, as examples of interconnected power systems, are investigated in this paper. Afterwards, it focuses on the inter-area optimization of the voltage and reactive power. Instead of performing the studies around one particular optimization, different aspects of the mathematical formulation of the optimization problem in the interconnected power system, including objective function, constraints and appropriate modeling of neighboring areas, are revisited more in depth. Various possible implementations of coordinated approaches, including centralized and decentralized structures, as well as non-coordinated approach in collaborative and non-collaborative environments are studied. In this respect, new contributions are proposed in this paper by using the distributed slack bus model and the limitation of the voltage and reactive power in the interconnections links. The comparative analysis between the available and proposed methods are discussed in terms of sub-optimality and time to convergence. The discussions are based on New England 39 bus system and the presented results in the literatures.
\end{abstract}

(c) 2013 Elsevier Ltd. All rights reserved.

\section{Introduction}

Voltage and reactive power control service is a critical ancillary service used by all system operators for secure and reliable operation of power systems. In a deregulated power system environment, appropriate provision of the reactive power support and voltage control services are among the major challenging responsibilities of the system operator. In this respect, the main challenges are due to numerous physical constraints, lack of transparent procurement and remuneration policies, and possibilities for discriminatory actions with respect to different resources [1]. Moreover, unlike the active power ancillary services (frequency control reserves), the reactive power cannot be transmitted efficiently through long distances because it leads to additional active and reactive power losses. As a result, the voltage has to be controlled by using special devices dispersed throughout the system. Hence, the system operators usually provide the voltage control services from the resources within their own controlled area.

Although the voltage control is primarily a local problem, the recent two decades widespread blackouts have demonstrated that the voltage instability and collapse could be considered as important as thermal overloads in major power outages worldwide [2]. It

\footnotetext{
* Corresponding author. Tel.: +41 2169346 61; fax: +41216934662.

E-mail address: omid.alizadeh@epfl.ch (O. Alizadeh Mousavi).
}

may involve several areas in the interconnected system and increase the scale of blackouts and even affect the intact areas [3]. The interconnected system, also referred as Multi-Area Power System (MAPS), involves several independent Transmission System Operators (TSOs) that each TSO corresponds to an area. The MAPS without inter-area voltage coordination may be operated in a nonoptimum state which means less security margin. For instance, the reference [4] demonstrates that in a MAPS, the optimization solution of a TSO for reducing the active power losses in its own region might lead to increase of losses in the interconnected area. Moreover, automatic and non-coordinated response of the voltage controllers may lead to unacceptable reactive power flow or voltage level in the control area or even in its neighbors. Hence, a wide coordinated control within control areas will bring additional value by proposing a global optimum solution. For this purpose, different TSOs must develop coordinated voltage control schemes inside its own area and between control areas to obstacle the voltage control concerns. However, up to now, each TSO only developed a specific voltage and reactive power regulation for its own area. These regulation schemes can be classified into: (a) hierarchical voltage regulation and (b) Centralized Voltage Regulation (CVR).

The hierarchical voltage regulation is a common classification which is usually implemented in three levels; primary, secondary and tertiary voltage regulation [5]. The Primary Voltage Regulation 


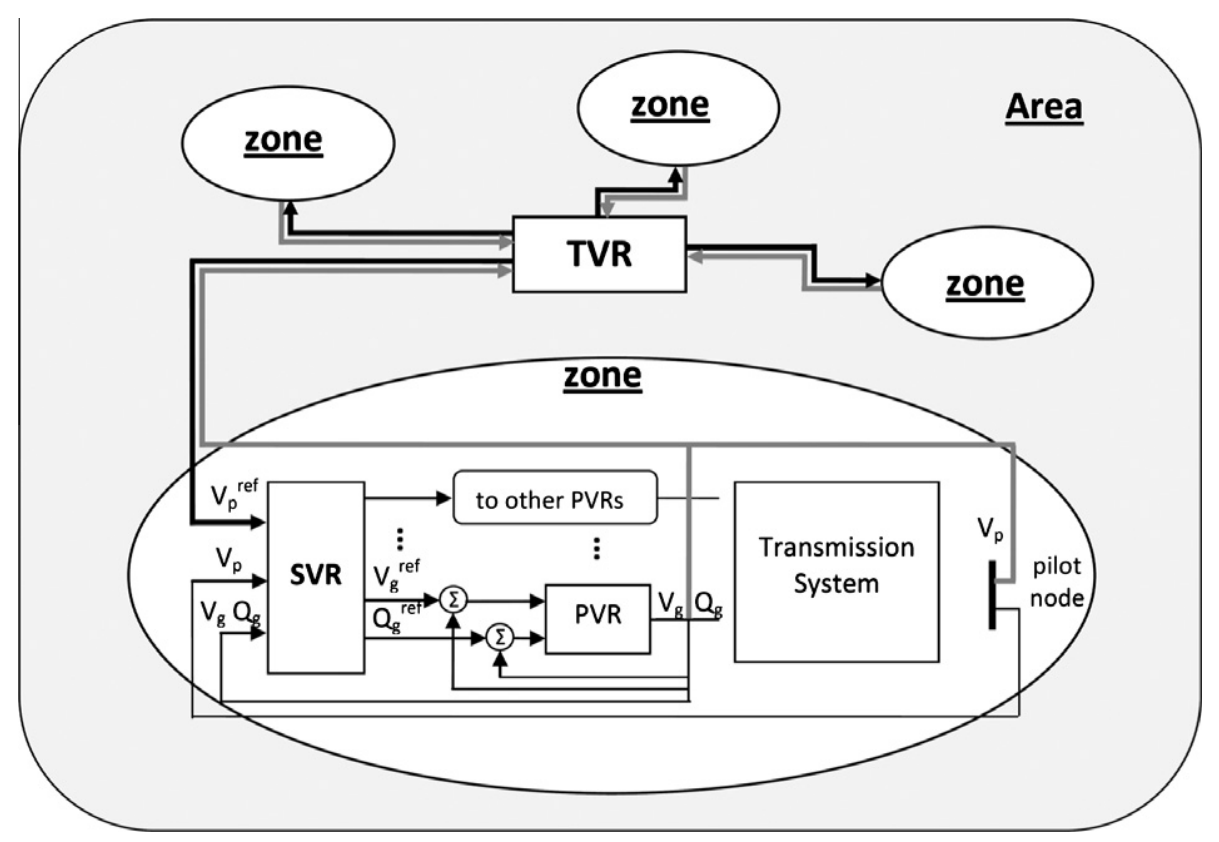

Fig. 1. Structure of a typical hierarchical voltage regulation with SVR and TVR.

(PVR) refers to the local response of the controllers (mainly generators). The voltage control at a zonal level is related to a Secondary Voltage Regulation (SVR) whereas the Tertiary Voltage Regulation (TVR) is at regional or national system level (area) [6]. However, in the centralized regulation, the voltage control is divided only into two classes: primary and Centralized Voltage Regulations (CVRs) [7]. In this case a CVR adjusts the set points of primary controllers from a control center. The definition, the implementation and the control objective of the hierarchical and the centralized regulation vary from one TSO to another [8].

The generic schemes of the hierarchical and centralized regulations are illustrated in Figs. 1 and 2, respectively. The depicted control scheme in Fig. 1, contains a TVR as a system control center which determines the optimal voltage set-points for the pilot buses based on a given optimization criterion applied to the whole system. These set-points are fed to the SVRs and they are used by local voltage/reactive power regulators, which are PVRs. A SVR controls the voltage at the given pilot bus(es) in such a manner that the generation of the reactive power is uniformly distributed among controllers inside the zone. Fig. 2 represents a typical structure of the centralized control, where the CVR determines the set-points of the voltage/reactive power regulators (PVRs) based on an optimization criterion. Despite these efforts for Single Area Power System (SAPS), the coordinated voltage control in the context of interconnected power systems has received less attention.

Current practices of different TSOs in ENTSO-E and NERC for the voltage and reactive power control and its provision and remuneration are studied in [3]. Table 1 summarizes the aforementioned practices. The corresponding provision and remuneration methods are provided in Table 2 .

Tables 1 and 2 demonstrate the intention of different system operators toward implementation of more sophisticated schemes

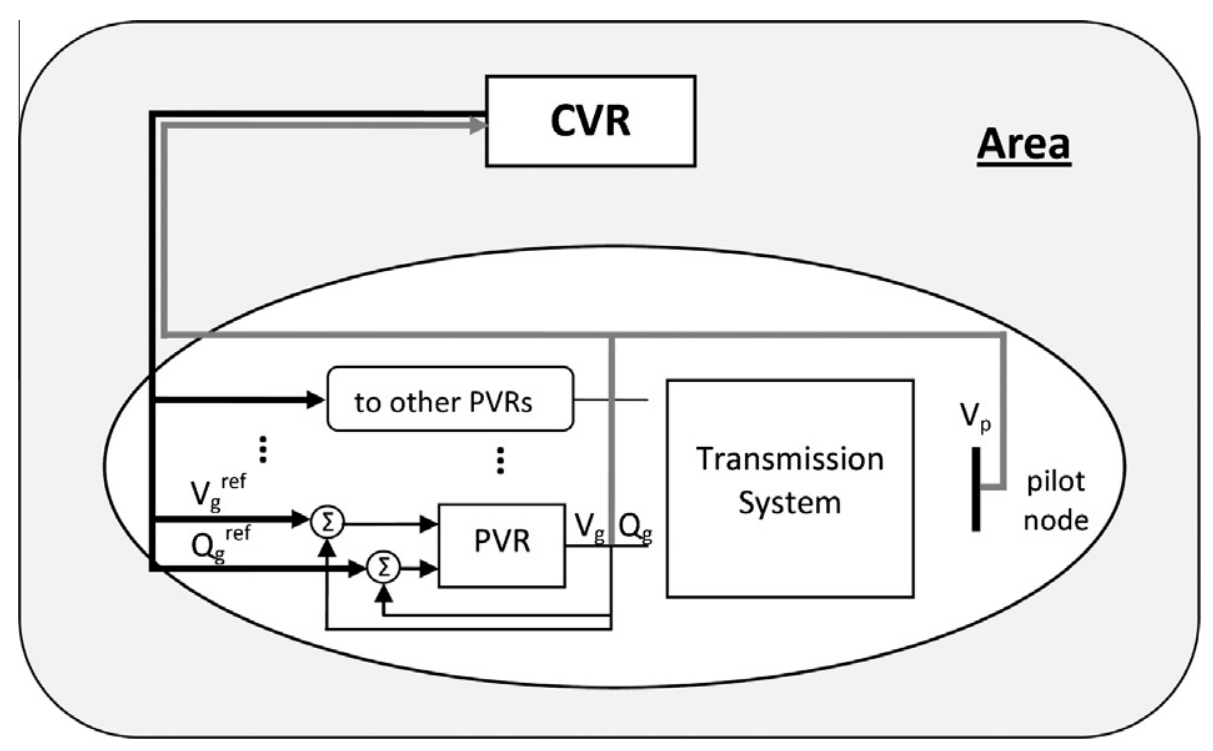

Fig. 2. Structure of typical Centralized Voltage Regulation (CVR). 
Table 1

Summary of different practices in voltage and reactive power control.

\begin{tabular}{|c|c|c|c|c|c|c|c|}
\hline \multirow[t]{3}{*}{ System } & \multirow[t]{3}{*}{ TSOs } & \multirow{2}{*}{\multicolumn{2}{|c|}{ Centralized }} & \multicolumn{4}{|c|}{ Hierarchical control } \\
\hline & & & & \multicolumn{2}{|l|}{ SVR } & \multicolumn{2}{|l|}{ TVR } \\
\hline & & $\begin{array}{l}\text { Voltage } \\
\text { control }\end{array}$ & $\begin{array}{l}\text { Reactive power } \\
\text { control }\end{array}$ & $\begin{array}{l}\text { Voltage } \\
\text { control }\end{array}$ & $\begin{array}{l}\text { Reactive power } \\
\text { control }\end{array}$ & $\begin{array}{l}\text { Voltage } \\
\text { control }\end{array}$ & $\begin{array}{l}\text { Reactive power } \\
\text { control }\end{array}$ \\
\hline France & RTE & & レ & & レ & & \\
\hline Italy & ENEL & & $\boldsymbol{\nu}$ & $\boldsymbol{r}$ & $\boldsymbol{r}$ & & \\
\hline \multirow[t]{3}{*}{ Belgium } & Elia & & & & & & \\
\hline & Practice & $\boldsymbol{r}$ & レ & & & & \\
\hline & Proposed & & & & レ & レ & レ \\
\hline Switzerland & Swissgrid & $\boldsymbol{V}$ & & & & & \\
\hline \multirow[t]{3}{*}{ Spain } & REE & & & & & & \\
\hline & Practice & $\boldsymbol{\nu}$ & & & & & \\
\hline & Proposed & & & レ & レ & レ & レ \\
\hline Germany & $\begin{array}{l}\text { Vattenfal, EON, RWE, } \\
\text { EnBW }\end{array}$ & & & & & & \\
\hline \multicolumn{8}{|l|}{ NORDEL } \\
\hline Netherlands & Tennet & & & & & & \\
\hline PJM & NERC & レ & $\boldsymbol{\nu}$ & & & & \\
\hline
\end{tabular}

Table 2

Summary of different provision and remuneration methods in voltage and reactive power control.

\begin{tabular}{|c|c|c|}
\hline System & Provision & Remuneration \\
\hline France & Long term bilateral contract with generators & $\begin{array}{l}\text { It is only specified for sensitive geographical zones. Energy generators at } \\
\text { fix rate }(€ / M V a r / h) .50 \% \text { increase if generator participates in SVR }\end{array}$ \\
\hline Italy & Hierarchical voltage control & Excess reactive energy withdrawal should pay ( $€ /$ MVar/h) \\
\hline Belgium & $\begin{array}{l}\text { Tender for providing voltage control resources based on price and } \\
\text { location of generating units }\end{array}$ & $\begin{array}{l}\text { The producers are paid for the actual consumed or generated reactive } \\
\text { power }(€ / \mathrm{MVar} / \mathrm{h})\end{array}$ \\
\hline Switzerland & $\begin{array}{l}\text { Day ahead reactive power planning, and bilateral agreement for } \\
\text { enhanced reactive power support }\end{array}$ & The provided reactive energy is compensated by ( $\mathrm{CHF} / \mathrm{MVar} / \mathrm{h})$ \\
\hline Spain & $\begin{array}{l}\text { For reactive power generation day ahead reactive planning, and voltage } \\
\text { control ancillary service in annual and daily time scopes. Both through } \\
\text { tendering process }\end{array}$ & $\begin{array}{l}\text { Monthly payment for both production and absorption considering; ( } 1) \\
\text { utilized reactive power ( } € / \text { MVar/h), (2) availability of additional band ( } € / \text { MVar) }\end{array}$ \\
\hline \multirow[t]{2}{*}{ Germany } & 1 - Bilateral agreements between concerned parties & 1 - Opportunity cost has to be included \\
\hline & 2 - Supplementary reactive power support in daily operational planning & 2 - Financial compensation based on bilateral agreements \\
\hline \multirow[t]{2}{*}{ NORDEL } & 1 - Generators compensatory reactive power supply & 1 - Without financial compensation \\
\hline & 2 - Additional reactive power supply & 2 - Yearly negotiation between system operator and producer \\
\hline Netherlands & Bilateral contracts with local generators & They are only paid for the reactive capacity not for reactive energy \\
\hline PJM & Compulsory basic voltage control & $\begin{array}{l}\text { The generators remunerated based on a regulated price including fixed } \\
\text { (\$/Month) plus opportunity cost }\end{array}$ \\
\hline
\end{tabular}

like centralized and hierarchical voltage controls. Thus, different approaches and practices from one TSO to another highlight the complexity of the interactions between the different system operations. In addition, the issue of additional coordination in MAPS is important because TSOs' accessibility to the neighboring TSOs' information is limited. This issue could become even more significant whenever the operating limits are reached and control efforts are saturated in an area [9]. Also, as it is mentioned before, a local voltage control problem may spread in MAPS and affect the intact areas. Therefore, a higher level coordination in MAPS is necessary for security of the power system. There are little relevant works to define required additional coordination between TSOs; and so on the voltage and reactive power control in MAPS need more attention by using centralized and decentralized control schemes.

Therefore, the issue of multi-area voltage and reactive power regulation (MAVR) could be considered between neighboring areas in interconnected power systems. Here, the investigations are performed on the optimization of the voltage and reactive power controllers in the operational planning stage. The obtained conclusions could be useful for the other design stages as well.

This paper is organized as follow. Section 2 focuses on the interarea voltage and reactive power management from two points of view: (a) the current practices of ENTSO-E and NERC, (b) the proposed methodologies in literatures. Then, the optimization methods are classified based on several criteria like collaborative/ non-collaborative behavior of TSOs and different coordination approaches including centralized/decentralized. A unified mathematical formulation is proposed for various optimization approaches. The distributed slack bus model and the reactive power limits in the interconnection links are proposed as original contributions in this work to improve the optimization methods. Section 3 presents in depth the advantages and drawbacks of the different optimization approaches for the voltage and reactive power management in the MAPS. The case studies and discussions on practical use/implementation of results are provided in Section 4.

\section{Optimization of the voltage and reactive power}

The survey of the current practices in Section 1 demonstrates the significance of the inter-area voltage coordination, although the provision of voltage control services are local. In this respect, the ENTSO-E operational handbook [9] recommends that interconnected TSOs should coordinate their actions and agree on an acceptable voltage range at each interconnection link, which can be roughly formulated as a zero reactive power flow at every interconnection link [10]. However, No reactive power flow at the interconnection links is difficult to apply. Observations show that reactive power flows are rarely negligible at the interconnection links [11]. 


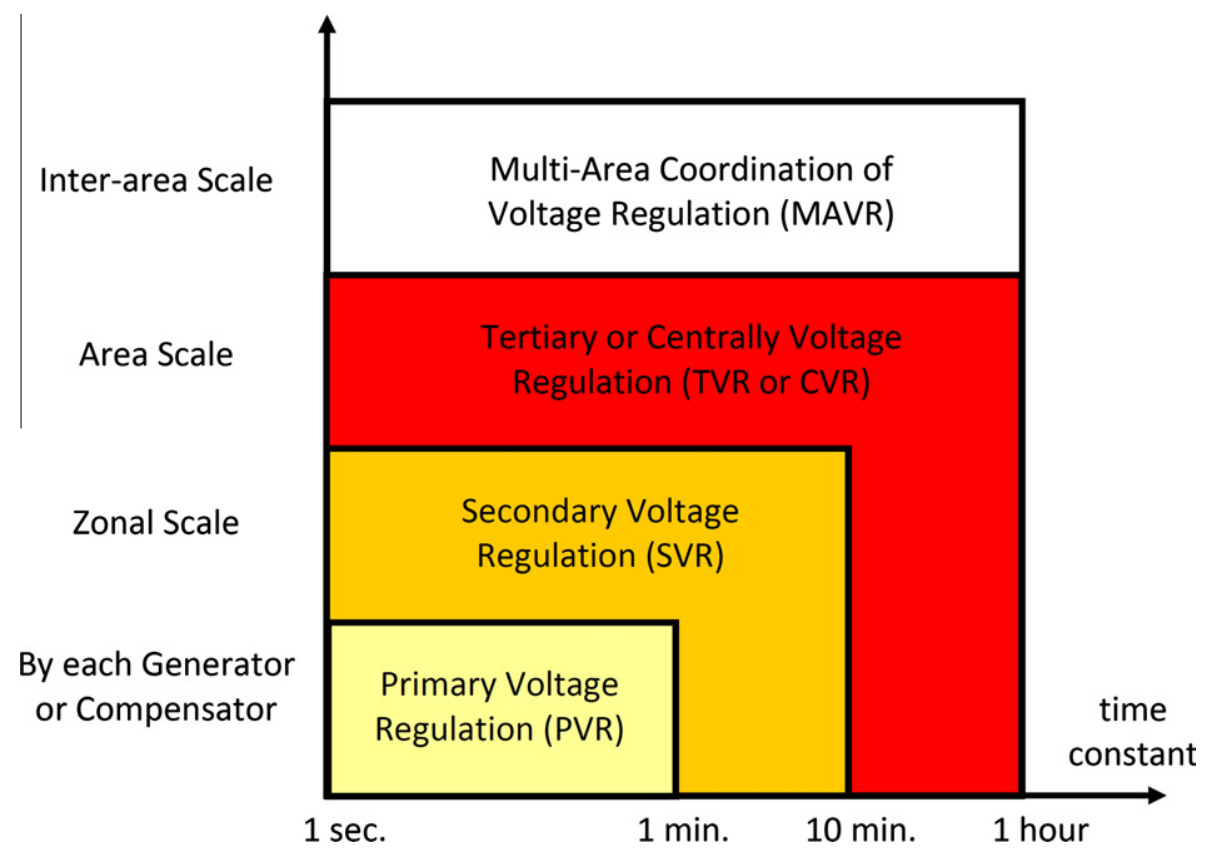

Fig. 3. Structure and time constant of the multi-area voltage regulation in comparison with the different level of voltage regulation [11].

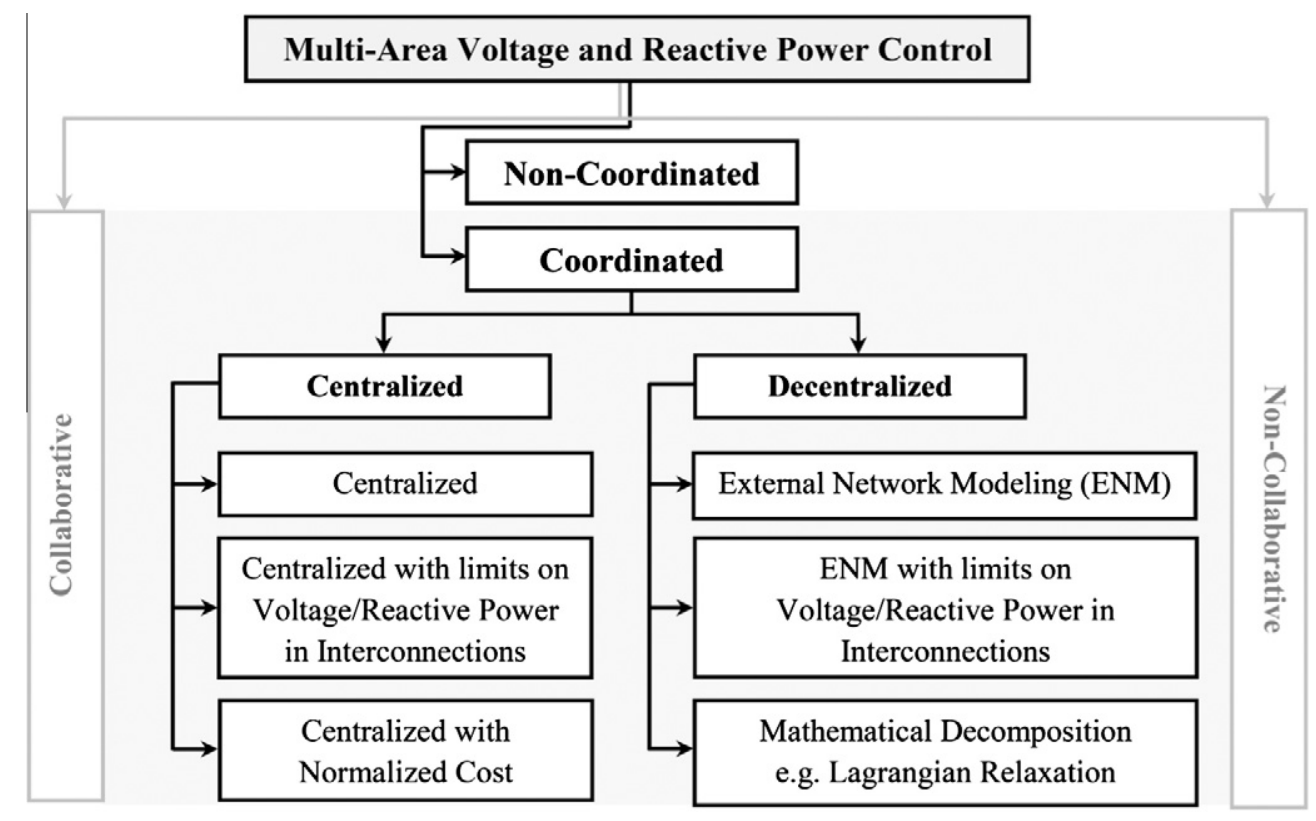

Fig. 4. Classification of the MAVR based on different coordination approaches and collaborative/non-collaborative behavior of TSOs.

The coordinated voltage and reactive power control in MAPS has been investigated in the literatures [10,12-17]. A new layer of hierarchical control to coordinate long-term control actions over several control areas in normal operating conditions is proposed in [11]. The corresponding time horizon for the proposed MAVR is shown in Fig. 3, in comparison with the different levels of hierarchical voltage regulation (PVR, SVR, and TVR). The absence of the MAVR and non-coordinated operation of the system would result into the responses of PVR and SVR, that it reduces reactive power reserve and consequently security margin.

The various MAVR approaches are classified based on the coordination and collaboration between TSOs, as shown in Fig. 4. Two main trends are developed for the MAVR coordination namely centralized and decentralized manners. These two optimization approaches and the corresponding formulations are explained more in depth in the following sub-sections. Note that these approaches could be implemented according to a collaborative or non-collaborative behavior of TSOs. More details on the advantages and disadvantages of these approaches are presented in Section 3.

In this respect the problem of interest, which is a general Optimal Power Flow (OPF) of a TSO (a single area), is formulated as follows.

$$
\begin{array}{cl}
\min _{u} & C(x, u) \\
\text { s.t } & h(x, u)=0 \\
& g(x, u) \leqslant 0
\end{array}
$$

where $C(x, u)$ is the objective function. $u$ and $x$ indicate the vector of control variables and the vector of the state variables, respectively. 
The equality constraints, given by $h(x, u)$, correspond to the power flow equations. The inequality constraints such as transmission line flow limits and generators capability limitations as well as node voltage limits are given by $g(x, u)$.

The system operator usually has to manage its reactive power resources for a specified active power dispatch $\left(P_{i}^{0}\right)$ obtained from the active power market. Therefore, in this optimization the control variables are the voltage of PV nodes and the reactive power of PQ nodes where control devices are connected to. These elements could be generators, transformers tap changer, synchronous condenser, capacitor banks, static VAR compensators and FACTS devices.

Moreover, literatures $[18,19]$ show that distributed slack bus model gives a better model for the response of the generators to the active power imbalances (here active power losses). Thus, no assumption is made a priori about the slack bus being unique or distributed. Any generating unit can play role in active power losses without introducing a set of participation factors. Therefore, the total injected power of generator $i$, from the set of generators $\left(\Omega_{G}\right)$, is decomposed into a constant term $\left(P_{i}^{0}\right)$, specified beforehand in the active power market, and a variable power $\left(P_{i}^{\text {Loss }}\right)$ representing its unknown contribution to the active power losses [18], as shown in equation

$P_{i}=P_{i}^{0}+P_{i}^{\text {Loss }} \quad i \in \Omega_{G}$

Each TSO has provided a particular formulation for its objective function $C(x, u)$ according to its network structure and specific requirements. Usually, a multi-objective function represents the objective function of one area. For instance, a general $C(x, u)$ can be written in the following form.

$O F=\omega_{1} \cdot \sum_{i \in \Omega_{G}} Q_{i}^{2}+\omega_{2} \cdot \sum_{i \in \Omega_{G}} P_{i}^{\text {Loss }}+\omega_{3} \cdot \sum_{i \in \Omega_{B}}\left(V_{n}-V_{n}^{s p c}\right)^{2}$

where $\Sigma\left(Q_{i}\right)^{2}$ is the quadratic sum of reactive power injection (QSQ), ${ }^{1} \sum\left(P_{i}^{\text {Loss }}\right)$ is the total active power losses, and $\sum\left(V_{n}-V_{n}^{s p c}\right)^{2}$ is the voltage deviation from a specified value or the voltage profile. Here, all voltage reference values $\left(V_{n}^{s p c}\right)$ are set to 1 p.u. $\omega_{i}$ are the weight coefficients of the corresponding objectives. $\Omega_{G}$ and $\Omega_{B}$ are the set of generators and buses, respectively. Other control objectives like effective reactive power reserve [20] and voltage stability criteria [21] could also be considered.

The objective function is subjected to the equality and inequality constraints given by (6)-(11). The active $(P)$ and reactive $(Q)$ power balance equality constraints are given by (6) and (7), respectively. The generators active power injection is considered according to (4). The transmission lines capacity limits are considered with (8). The limits of the voltage magnitudes and their phase angles for each bus are given by (9) and (10), respectively. The generators reactive power injection limits are considered with (11). The generators participation in the active power losses is limited by (12). The maximum reactive power support of a generator is obtained considering the limitation of the field current (13) and the limitation of the armature current (14). Literatures show that the explicit representation of generators terminal voltages on generators capability limits plays a dominant role [22].

$P_{i n}^{0}+P_{i n}^{\text {Loss }}-P_{d n}-\sum_{m \in \Omega_{B}} V_{n} V_{m}\left(G_{n m} \cos \theta_{n m}+B_{n m} \sin \theta_{n m}\right)=0 \quad n \in \Omega_{B}$

\footnotetext{
${ }^{1}$ Quadratic objective works better than absolute one, since it is well known in the case of quadratic optimization methods. Also, there is similarity between the quadratic objective and the reactive power cost function. Besides, all generators that participate in the voltage and reactive power control could be remunerated according to their participation, whether reactive power generation (injection) or consumption (absorption).
}

$Q_{i n}-Q_{d n}-\sum_{m \in \Omega_{B}} V_{n} V_{m}\left(G_{n m} \sin \theta_{n m}-B_{n m} \cos \theta_{n m}\right)=0 \quad n \in \Omega_{B}$

$\left(G_{n m}^{2}+B_{n m}^{2}\right) \cdot\left(V_{n}^{2}+V_{m}^{2}-2 V_{n} V_{m} \cos \theta_{n m}\right) \leq\left(T_{l}^{\max }\right)^{2} \quad\{n, m\} \in l, l \in \Omega_{L}$

$V_{n}^{\min } \leq V_{n} \leq V_{n}^{\max } \quad n \in \Omega_{B}$

$-\pi \leq \theta_{n} \leq \pi \quad n \in \Omega_{B}$

$Q_{i}^{\min } \leq Q_{i} \leq Q_{i}^{\max } \quad i \in \Omega_{G}$

$0 \leq P_{i}^{\text {Loss }} \leq\left(P_{i}^{\max }-P_{i}^{0}\right) \quad i \in \Omega_{G}$

$Q_{i}^{\max }=\min \left\{\begin{array}{l}\bar{Q}_{r i}=-\frac{V_{i}^{2}}{X_{S i}}+\sqrt{\frac{V_{i}^{2} \bar{I}_{f t}^{2}}{X_{S i}^{2}}-P_{i}^{2}} \\ \bar{Q}_{a i}=\sqrt{V_{i}^{2} \cdot \bar{I}_{a i}^{2}-P_{i}^{2}}\end{array}\right.$

In this formulation $\Omega_{L}$ is the set of lines. $n$ and $m$ are the index of buses, $l$ is the index of lines, $i$ is the index of generators, and $d$ is the index of demands. $V_{n}$ is the voltage magnitude of bus $n$, and $\theta_{n m}$ is the voltage angle difference between the buses $n$ and $m$. $G_{n m}$ and $B_{n m}$ are the real and imaginary part of the $\{n, m\}$ element of the admittance matrix. The vector $P_{i}^{\text {Loss }}$ models the distributed slack bus for compensating the active power losses of the transmission system. This modeling allows every generator to participate in the system active power losses. Reactive power capacity limit of each generator are specified by $Q_{i}^{\min }$ and $Q_{i}^{\max }$. The limits of voltages at bus $n$ are $V_{n}^{\min }$ and $V_{n}^{\max }$. The maximum transferred power of the transmission lines is given by $T_{l}^{\max }$. In (13) and (14) $V_{i}$ is the generator terminal voltage, $P_{i}$ is the generator active power output, $\bar{I}_{f i}$ is the maximum field current, $\bar{I}_{a i}$ is the maximum armature current, and $X_{s i}$ is the synchronous reactance.

It must be mentioned when a single central control satisfies a certain objective function for the interconnected system; the result would be the system wide optimal solution. However, in the multiTSO system, where there are several entities, specifying a certain optimization for the whole system is challenging. Generally, the optimization in the multi-TSO system is referred to the centralized optimization. Different aspects of the centralized optimization of MAPS are investigated in following sub-section.

\subsection{Centralized optimization}

A centralized control scheme addresses the multiple areas as one contiguous area with multiple parties. Thus, an optimization problem over all areas is solved by a single central controller (CC) [12]. In this respect, it is assumed that each $\mathrm{TSO}_{k}$ has its own objective function $C_{k}(x, u)$ and constraints $h_{k}(x, u)$ and $g_{k}(x, u)$. The objective of each TSO can be selected with different combinations of the multi-objective function in (5). Note that the TOSs can provide their objectives and constraints information to the control center (CC) either in a collaborative or non-collaborative manner. The multi-objective optimization for NTSO number of TSOs could be represented by (15) subject to the constraints in (2) and (3) [13].

$\min _{u}\left[C_{1}(x, u), \ldots, C_{k}(x, u), \ldots, C_{\mathrm{NTSO}}(x, u)\right]$

Generally, the objective function of the centralized optimization is presented as the sum of all TSOs cost functions $\sum_{k \in \mathrm{NTSO}} C_{k}(x, u)$. This optimization defines the system wide optimal solution. The main concern of the centralized optimization is the effort of the TSOs to fulfill the individual and possibly conflicting objectives. Thus, the objective of a single TSO may adversely affect other TSOs 
[14]. For this issue, a compromise method is proposed in [13] to obtain the Pareto-front solution. In this method that is called centralized with Normalized Cost (NC), each TSO solves an initial optimization to minimize its own cost function while respecting all constraints in the system. For the $k$ th TSO, the obtained initial solution is given by $u_{k}^{*}$. Then, another optimization minimizes the distance of final solution from the obtained initial solutions in a normalized multi-dimensional space, as shown in

$\min _{u} \sum_{k \in \mathrm{NTSO}}\left(\bar{C}_{k}(u)-\bar{C}_{k}\left(u_{k}^{*}\right)\right)^{2}$

where $\bar{C}_{k}(u)$ is the normalized cost function of area $k$, given by (17).

$\bar{C}_{k}(u)=\frac{C_{k}(u)}{C_{k}^{0} \times \chi_{k}}$

In this formulation $C_{k}^{0}$ and $\chi_{k}$ are given by (18) and (19), respectively. $C_{k}^{0}$ is the average of the overcost supported by $\mathrm{TSO}_{k}$ for the other NTSO areas. $\chi_{k}$ penalizes the detrimental impact of $\mathrm{TSO}_{k}$ on the other TSOs.

$C_{k}^{0}=\sum_{j \in \mathrm{NTSO}} \frac{C_{k}\left(u_{j}^{*}\right)-C_{k}\left(u_{k}^{*}\right)}{\mathrm{NTSO}}$

$\chi_{k}=\sum_{j \in \mathrm{NTSO}} \frac{C_{j}\left(u_{k}^{*}\right)-C_{j}\left(u_{j}^{*}\right)}{C_{j}^{0}}$

The objectives are normalized to ensure that the solution of the problem has some properties of fairness. The fairness of the solution is studied in the economic sense by some criteria, namely efficiency, accountability and altruism. The proposed scheme is quite effective in collaborative strategy of TSOs. Moreover, it is shown that this scheme is not robust (see Section 4.3) to biased information of TSOs. Whenever they provide wrong information about their objectives and constraints, they may affect the allocation of resources in certain circumstances [13].

Although the centralized and the centralized with NC optimization procedures could define the system wide optimal and fair solutions, respectively, they suffer from several drawbacks. The first disadvantage is that the centralized solutions are susceptible to single point failure (centralized control center). Another difficulty to implement a centralized control in MAPS is due to the TSOs intention to not reveal their operational information to the other TSOs. Moreover, implementation of a wide area control scheme would be technically more expensive and requires more communication. Furthermore, some issues, like the fairness of the solution, provoke challenges when the TSOs have different objectives and constraints, specifically in the case of a non-collaborative environment. In addition to the aforementioned difficulties and limitations, a major reason to implement a decentralized control in MAPS comes from the facile adaptation to the current structurally decentralized control situation of the system.

\subsection{Decentralized optimization}

In the decentralized optimization scheme, the overall optimization in the interconnected system is divided into sub-problems according to the areas. Each TSO maintains its prerogatives and optimizes its own control area according a specific procedure [17]. In such cases, coordination is needed because the chosen setting of one area possibly influence the entire of the system and the choice of the setting of the other areas [23].

The non-coordinated reactive power scheduling in MAPS, when each TSO only solves its own optimization, increase the cost of the interconnected system as well as the cost of each area, specifically when neighboring TSOs apply conflicting objectives [24]. Even if the neighboring TSOs satisfy a same objective separately by their own, not only a communication would be necessary between the areas, but also the obtained results would be sub-optimal in comparison with the centralized one [25]. The coordination could be achieved by exchanging some information between neighboring areas.

In order to investigate the appropriate coordination in the MAPS, the decentralized optimization can be formulated as follow. Generally, every $\mathrm{TSO}_{k}$ confronts with the following optimization.

$$
\begin{aligned}
\min _{u_{k}} & C_{k}\left(x_{k}, u_{k}, \tilde{z}_{k}\right) \\
\text { subject to } & h_{k}\left(x_{k}, u_{k}\right)=0 \\
& g_{k}\left(x_{k}, u_{k}\right) \leqslant 0 \\
& h_{k-\varsigma}\left(x_{k}, u_{k}, \tilde{z}_{k}\right)=0 \\
& g_{k-\varsigma}\left(x_{k}, u_{k}, \tilde{z}_{k}\right) \leqslant 0
\end{aligned}
$$

where (21) and (22) are its own equality and inequality constraints. (23) and (24) depends on decision variables from the problems of the other areas and they are so-called complicating (or coupling) equality and inequality constraints, respectively. For each area, the number of complicating constraints depends on the set of interconnections $\left(\Omega_{k-c}\right) \cdot \tilde{z}_{k}$ represents the complicating variables which belongs to both $\mathrm{TSO}_{k}$ and $\mathrm{TSO}_{k^{\prime}}\left(k^{\prime} \neq k\right)$. The number of these border variables depends on the number of the buses that the interconnections are connected to. These optimizations cannot be solved separately for each area $k$, because $\tilde{z}_{k}$ is involved in the objective function (20) and/or in the complicating constraints (23) and (24) of more than one TSO. In other words, these variables and the subsequent constraints bring the need for coordination and communication.

Note that decentralized optimizations are iterative in nature. Additional subscript $t$ specifies the objectives and variables in $t$ th iteration. For instance $C_{k, t}$ refers to the objective function of $\mathrm{TSO}_{k}$ at iteration $t$. Here, this subscript is not shown in the formulation, except when it is needed, to avoid the complex formulation.

In general, there is no coordination between control areas. The lack of coordination can be eliminated by implementing different decomposition approaches [26]. Literatures [12,15,16,23,26] propose various decomposition approaches for the voltage and reactive power management in the MAPS. These approaches are divided into External Network Modeling (ENM) and mathematical decomposition methods.

\subsubsection{External Network Modeling}

Phulpin et al. [16] suppose that each TSO assumes an external network equivalent for its neighboring areas and solves its own optimization defined by (25) regarding its own constraints given by (21) and (22) and regardless to the neighboring systems' objectives. The external network equivalents are considered as equality constraints given by (26), where $\tilde{z}_{k}^{*}$ denotes the parameters of the external network equivalent. The superscript $*$ indicates the variables which they are calculated in previous iteration and they are kept constant in this iteration. Note that it is supposed that every TSO fairly use the same type of equivalent to compute the neighboring area's model at the interconnections.

$\min _{u_{k}} C_{k}\left(x_{k}, u_{k}\right)$

$h_{k-\varsigma}\left(x_{k}, u_{k}, \tilde{z}_{k}^{*}\right)=0$

Then, it is assumed that all TSOs apply the solution to their own systems as a part of the interconnected system and each TSO measures variables to set up the external network equivalents. Although the proposed scheme does not need any information exchange between TSOs, each TSO should communicate with a 
centrally control center which performs a power flow to determine the overall system state. It is possible that this power flow doesn't converge.

Similarly, a coordinated decomposition can be proposed for a specific time interval. For this, a center like TSC - TSO Security Cooperation - in Europe [27] could receive the calculated control actions of each TSO and perform a load flow to evaluate the state of the interconnected system. Then, the load flow results are sent back to each area. The load flow results in the interconnections could be considered as common knowledge of all TSOs. The TSOs improve their external network equivalents corresponding to the tie-line flows. Note that this load flow should be performed by distributed slack bus model according to the active power loss participation factors, obtained from the OPF of each area. The algorithm is repeated until convergence is reached $\left|C_{k, t}-C_{k, t-1}\right| \leqslant \varepsilon$. In this approach, each TSO applies a parameter fitting method on the load flow results in the interconnection to obtain the external network equivalents as follow [16].

$\min _{z_{k}} \sum_{t=1}^{T} \beta^{T-t} \times\left\|z_{k}-z_{k, t}^{*}\right\|^{2}$

In (27) $\beta \in[0,1]$ is a memory factor and $z_{k, t}^{*}$ is the parameter of the external network equivalent in $t$ th iteration. Here, $\beta$ is considered to be equal to 0.75 . The simulation results for $\beta=0$ swing as shown in [12]. Various external network models are studied in $[15,16]$ including PQ-equivalent, PV-equivalent, Thevenin-equivalent and more advanced models like REI equivalent and non-reduced power system equivalent. It is shown that PQ-equivalent could achieve better performance in terms of convergence and sub-optimality. It should be mentioned that in the first iteration, the interconnections are modeled by a fixed active power, obtained from a DC load flow. It can be deliberated as an agreed active power flow in the interconnections between neighboring TSOs.

\subsubsection{Mathematical decomposition methods}

Mathematical decomposition of optimizations is widely proposed in the literature $[12,23,26,28,29]$, based on Lagrangian, augmented Lagrangian, approximate Newton directions and primal dual interior point method. The decomposed sub-problems are then solved in an iterative way, independently but in coordinated way. Two coordination approaches based on Lagrangian methods at the existing or fictitious border buses [12] are: (a) the coordination via an adjustment at the interfaces (borders) to the neighboring areas, and (b) the coordination by exchange of variables which belong to neighboring areas. In these approaches the neighboring areas exchange the value of the border variables and the Lagrangian multipliers related to the complicating variables/constraints.

2.2.2.1. Decomposition based on adjustment at the border. In first approach each area independently solves a modified optimization that includes its own variables and the border variables $\left(\tilde{z}_{k}\right)$ shared with the other area. The coordination is attained via a coupling constraint, forcing the border variables to be equal [26]. By considering these coupling constraints into the objectives, the following optimization is reached subject to (21) and (22).

$$
\min _{u_{k}} C_{k}\left(x_{k}, u_{k}, \tilde{z}_{k}\right)+\sum_{k^{\prime} \neq k} \lambda_{k^{\prime}}^{*}\left(\tilde{z}_{k}-\tilde{z}_{k^{\prime}}^{*}\right)
$$

In this formulation $\lambda_{k^{\prime}}^{*}$ is the vectors of the Lagrangian multipliers corresponding to the border variable $\left(\tilde{z}_{k^{\prime}}^{*}\right)$ determined by the subproblems of the neighboring areas $\left(k^{\prime} \neq k\right)$.

In fact in this approach, all TSOs with shared borders calculate the corresponding complicating border variables $\left(\tilde{z}_{k}\right)$. Then, TSOs use the observations from borders to model the influence of other areas on their own areas.

2.2.2.2. Decomposition based on passing adjacent variables. In the second approach, the coordination is attained through exchanging some dedicated variables between neighboring areas. The subproblem of $\mathrm{TSO}_{k}$ is obtained by accounting the complicating constraints of the foreign areas $\left(h_{k^{\prime}-\varsigma}\right.$ and $\left.g_{k^{\prime}-\varsigma}\right)$ and adding them to the objective function while maintaining its own complicating constraints $[23,26,28]$. Therefore, $\mathrm{TSO}_{k}$ solves the following optimization.

$$
\begin{aligned}
& \min _{u_{k}} C_{k}\left(x_{k}, u_{k}, \tilde{z}_{k}, \tilde{z}_{k^{\prime}}^{*}\right)+\sum_{\Omega_{k^{\prime}-\varsigma}} \lambda_{k^{\prime}-\varsigma}^{*} h_{k^{\prime}-\varsigma}\left(x_{k}, u_{k}, \tilde{z}_{k}, \tilde{z}_{k^{\prime}}^{*}\right) \\
& +\sum_{\Omega_{k^{\prime}-\varsigma}} \mu_{k^{\prime}-\varsigma}^{*} g_{k^{\prime}-\varsigma}\left(x_{k}, u_{k}, \tilde{z}_{k}, \tilde{z}_{k^{\prime}}^{*}\right)
\end{aligned}
$$

subject to (21) and (22) as well as following constraints.

$h_{k-\varsigma}\left(x_{k}, u_{k}, \tilde{z}_{k}\right)=0: \lambda_{k-\varsigma}$
$g_{k-\varsigma}\left(x_{k}, u_{k}, \tilde{z}_{k}\right) \leq 0: \mu_{k-\varsigma}$

In this formulation, the second and the third part of (29) demonstrate the complicating constraints of the other sub-problems $\left(k^{\prime}-\right.$ $\neq k$ ) as relaxed constraints in the objective function. (30) and (31) give the complicating constraints of the sub-problem $k$ as hard constraints. $\lambda_{k-\varsigma}$ and $\mu_{k-\varsigma}$ are the Lagrangian multipliers obtained from the solution of the sub-problems $k$. They could be interpreted as the cost of providing power from the neighboring areas.

The coupling constraints could be the power balance equations at the existing or fictitious border buses. In $[28,30]$ these coupling constraints are the interconnections active and reactive power flow equations $\left(h_{k-\varsigma}\right)$ but not their power flow limits $\left(g_{k-\varsigma}\right)$.The active and reactive power flows from bus $n$ to bus $m$ at the interconnection $\varsigma$ are calculated using (32) and (33), respectively.

$P_{n m-\varsigma}=V_{n}^{2} G_{n m}-V_{n} V_{m} G_{n m} \cos \theta_{n m}-V_{n} V_{m} B_{n m} \sin \theta_{n m}$

$Q_{n m-\varsigma}=-V_{n}^{2}\left(B_{n m}-\left(B_{s h} / 2\right)\right)+V_{n} V_{m} B_{n m} \cos \theta_{n m}-V_{n} V_{m} G_{n m} \sin \theta_{n m}$

where $B_{s h}$ is the tie-line shunt susceptance. It must be noted that if several tie-lines are connected to a bus, $P_{n m-\varsigma}$ and $Q_{n m-\varsigma}$ are the sum of all tie-lines power flows connected to that bus.

The interconnections power flow tolerances calculated on both sides are used as convergence criteria. Here, any specific treatment is needed to define the reference bus, since a distributed slack bus is utilized for the active power losses. Besides, the initial values of parameters in the first iteration are equal to zero except the voltages which are equal to one.

\subsubsection{Implementation and evaluation of the decentralized optimization}

Every decomposition approach can be implemented in a sequential way or in a synchronized way [12]. In the synchronized way, also referred as parallel way, every TSO solves its own optimization simultaneously and then exchange the variables. In the sequential way, TSOs solve their optimizations after each other by updating the variables of the neighboring areas obtained from their previous solutions. The sequential approach has the disadvantage of being slower than the parallel one. On the other hand, the synchronized approach may not keep the system inside its feasible operating region at every moment since each TSO ignores the other TSOs control actions. This infeasibility could be due to the non-convergency of the TSO's OPF or load flow (in the case of ENM). In these cases, additional coordination should be designated to bring the solution back inside the feasible region [31]. For instance, Ref. [28] introduces the fictitious reactive power sources 
in order to obtain feasibility of nodal reactive power balance. These fictitious sources are added in the buses with reactive loads and the sum of square of them is added in the objective function with a high cost (reactive load shedding). This method could be used for both decentralized approaches with ENM and Lagrangian Relaxation (LR).

The result of the decentralized optimization can be evaluated based on the distance to the solution of the centralized optimization $[11,17]$. This is usually referred as sub-optimality or additional cost of the decentralized operation. Besides, the required number of iterations, or literally the required communication, to obtain the optimal solution could be considered as another criterion to evaluate the decentralized optimization scheme.

One of the main specifications of the decentralized approach in comparison to the centralized approach is that it deals with smaller problems in an iterative manner. Here, the complexity of the simulation time, results from the interaction between the number of iterations and the size of the problem. The simulation results in [28] particularly demonstrate that the decentralized approach is more time consuming when the power system size increases, while in the small test systems it is the contrary.

\section{Various approaches for collaborative optimization of the voltage and reactive power in MAPS}

The difficulties for the voltage and reactive power control in MAPS are discussed in Section 1. Then, general formulations for the voltage and reactive power control in SAPS and MAPS are presented in Section 2. This section classifies various approaches that could be proposed for the collaborative coordination of the voltage and reactive power control in MAPS. Advantages and disadvantages of these approaches are investigated more in depth.

It is worth to remind the assumptions taken into consideration here:

- The optimization variables are voltage/reactive power of generators, voltage magnitudes and voltage phase angles of buses, and active power losses of generators.

- Distributed slack bus models the generators responses to the active power losses. This modeling improves the optimization results in comparison with single slack bus, specifically in high loading levels.

- The generators active power output is composed of a fixed and a variable term. The former is known through active power market and the latter changes by a distribution of losses among the generators.

- The TSOs work in a collaborative frame work and they do not provide biased information. The effect of non-collaborative strategies including biased formulation of the constraints and objectives on various approaches will be studied in Section 4.

- AC power flow is calculated using distributed slack bus with a given participation factor obtained from the result of OPF in each area.

- The variables at the other side of the interconnection are assumed as the border variables.

- In this way, the interconnections belong to both areas. Thus, it is not needed to define a dummy bus in the middle of each tie-line to identify the border variables.

- For decentralized optimization approaches, TSOs solve their own optimizations in a synchronized way. That means at each iteration, all areas perform their own optimization concurrently and then the exchange information between areas are updated.
The different approaches implemented in this paper are explained below.

(a) Non-coordinated: Each TSO solves its own sub-problem and implement the solution without further coordination. A power flow determines the state of the system for the obtained control actions. The main features of the non-coordinated operation are higher amount of sub-optimality and possible infeasibility in the sub-problems or the load flow due to conflictual decisions.

All of the next approaches are coordinated.

(b) Centralized: All TSOs send their objectives and constraints to a center. This center combines information and solve an optimization for the entire system. The obtained solution is considered as the Utopian optimum or the system wide optimal solution. This solution is not necessarily fair for all TSOs.

(c) Centralized - Normalized Cost (NC): This approach solves $(\mathrm{NTSO}+1)$ optimization problems to obtain control actions using the formulation presented by (15)-(19). Note that there is a distance between the solution of the Centralized and the Centralized NC that is additional cost to obtain a fair solution. This additional cost in long term can lead TSOs to improve the voltage control in their own areas.

(d) Decentralized - External Network Modeling (ENM): Every TSO solves its own problem with a PQ model for each interconnection. The obtained control actions are sent to a center and a power flow calculates the whole system state. The results at the interconnections are sent back to areas to update the model of neighboring systems using (27), iteratively. In general the solution suffers from sub-optimality.

(e) Decentralized - Lagrangian Relaxation (LR): Every TSO solves its own problem by exchanging the border variables including the voltages magnitudes and phase angles as well as Lagrangian multipliers for the active and reactive power flow equality constraints on tie-lines. In comparison with Decentralized ENM, Decentralized LR has negligible suboptimality and it doesn't need the AC power flow to determine the system state, but more information and iterations are required.

One can propose to limit the reactive power flows on interconnections to localize more the reactive power provision within each area in order to increase the transfer capability of the interconnections. However, in this case the inter area reactive power support of neighboring areas will become more limited. In this respect, the objective function changes as follow.

$O F+\omega_{4} \cdot \sum_{\Omega_{k-\varsigma}}\left(Q_{n m-\varsigma}^{2}+Q_{m n-\varsigma}^{2}\right)+\omega_{5} \cdot \sum_{\Omega_{k-\varsigma}}\left(V_{n-\varsigma}-V_{m-\varsigma}\right)^{2}$

where the first term shown by $O F$ is the main objective function given in (5). The second term is the sum of the square of the reactive power flows at both ends of the interconnections. The last term is the square of the voltage differences between the interconnection ends. $\omega_{i}$ are weighting coefficients that should be selected in such a way to compensate the effect of different objective functions, and to minimize the total cost of the system. One may propose to add the reactive flow in the middle of interconnection to the objective function in order to minimize the transferred reactive power. However, in this case the results would be dependent on the interconnection model ( $\pi$-model and $T$-model). 
Reformulating the objective function in the form given by (34) could bring advantages for the Centralized and Decentralized ENM approaches. Thus, the following two approaches are investigated in addition.

(f) Centralized with additional limits on voltage/reactive power in the interconnections (Centralized LI): In the centralized approach, additional limits on voltage/reactive power in the interconnections reduce the effect of different (and even conflicting) objectives of neighboring TSOs on the others. In other words it can improve fairness of the centralized optimization. In non-collaborative environment it decreases the impact of conflictual decisions of the neighboring TSOs. However, the total cost becomes higher than the centralized approach. This additional cost is paid to oblige TSOs to provide their requested reactive power within their own area and it could be shared among TSOs. For the centralized optimization, the limits on the reactive power in the interconnections and the limits on the voltage in the interconnections work in the same direction, but the first is more effective than the latter $\left(\omega_{4} \neq 0, \omega_{5}=0\right)$. Here, the term effective means less reactive power flow in the interconnections while the objective functions maintain the same value by adjusting the weights in (34). The reason is that, the transmitted reactive power between areas is directly minimized in the objective function.

(g) Decentralized ENM with additional limits on voltage/reactive power in the interconnections (Decentralized ENM LI): Additional limits on voltage/reactive power in the interconnections in the decentralized ENM decrease the sub-optimality and the required communication. Furthermore, if the controller fails in one area the effects are more limited. For Decentralized ENM, the limits on the voltage in the interconnections result in lower total cost than the limits on the reactive power $\left(\omega_{4}=0, \omega_{5} \neq 0\right)$.
It is worth to mention that for the approaches which require power flow to determine the system state (Non-Coordinated and Decentralized ENM); the obtained voltage magnitudes may not satisfy their limits. It occurs more frequently in the first iterations as shown in [16].

These optimization approaches for MAVR are studied for the case study in the next section. The specifications, advantages and disadvantages of these approaches are discussed based on illustrative example as well as the results and conclusions in the literatures.

\section{Case Studies and discussions on practical use/ implementation of results}

The aforementioned approaches are evaluated on New England 39 bus system. The one line diagram of the system is represented in Fig. 5 and its description and data can be found in [20]. This system is partitioned into three areas, namely area A, B and C. The areas are selected such that at least one border bus (bus 4 and 14 ) is connected to more than one interconnection line and one border bus (bus 39) is connected to a generator. This particular system allows to verify the presented general formulation when there are different number of the complicating variables and constraints.

It is well-known that the reactive power generation effectively depends on the loading level in the system. In order to obtain more general conclusions, simulations are performed for two loading patterns with different quantities of loads namely high (7011.66 MW + 1620.22 MVAR) and low (6110 MW + 1409.70 MVAR). The simulations results provided in this section are carried out for the high and low loading patterns.

The approaches mentioned in Section 3 for MAVR are investigated with three different study cases when neighboring areas hold:

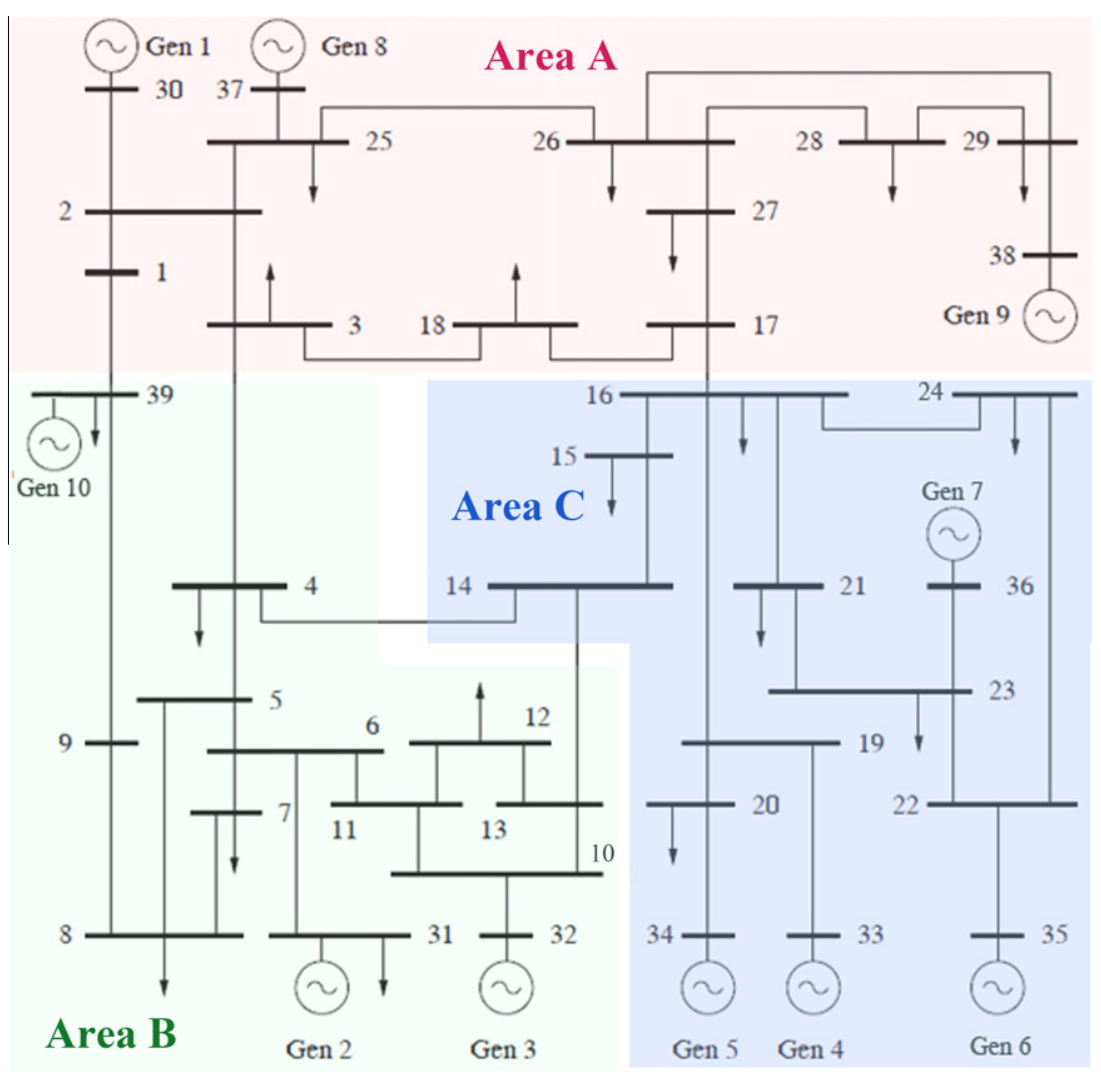

Fig. 5. New England 39-bus system with three areas. 
Table 3

The value of control variables for New England 39-bus system in high loading pattern when areas apply the same objective function.

\begin{tabular}{|c|c|c|c|c|c|c|c|c|c|c|c|}
\hline & & \multicolumn{3}{|l|}{ Area A } & \multicolumn{3}{|l|}{ Area B } & \multicolumn{4}{|l|}{ Area $\mathrm{C}$} \\
\hline & & $\mathrm{G}_{1}$ & $\mathrm{G}_{8}$ & $\mathrm{G}_{9}$ & $\mathrm{G}_{2}$ & $\mathrm{G}_{3}$ & $\mathrm{G}_{10}$ & $\mathrm{G}_{4}$ & $\mathrm{G}_{5}$ & $\mathrm{G}_{6}$ & $\mathrm{G}_{7}$ \\
\hline \multirow[t]{2}{*}{ Non-coordinated } & $V(\mathrm{pu})$ & 1.0155 & 1.0197 & 0.9854 & 1.0600 & 1.0600 & 1.0492 & 1.0600 & 1.0600 & 1.0528 & 1.0600 \\
\hline & $Q$ (MVAR) & 49.26 & 39.17 & 47.76 & 305.07 & 316.39 & 270.50 & 208.36 & 175.66 & 246.08 & 190.99 \\
\hline \multirow[t]{2}{*}{ Centralized } & $V(\mathrm{pu})$ & 1.0600 & 1.0600 & 1.0600 & 1.0365 & 1.0359 & 1.0467 & 1.0600 & 1.0600 & 1.0500 & 1.0600 \\
\hline & $Q$ (MVAR) & 131.35 & 63.05 & 92.73 & 243.67 & 245.55 & 223.04 & 187.53 & 164.13 & 214.24 & 182.62 \\
\hline \multirow[t]{2}{*}{ Centralized NC } & $V(\mathrm{pu})$ & 1.0600 & 1.0600 & 1.0600 & 1.0182 & 1.0190 & 1.0259 & 1.0557 & 1.0600 & 1.0425 & 1.0600 \\
\hline & $Q$ (MVAR) & 162.11 & 80.70 & 102.64 & 233.74 & 237.65 & 197.75 & 188.12 & 179.20 & 205.47 & 203.07 \\
\hline \multirow[t]{2}{*}{ Decentralized ENM } & $V(\mathrm{pu})$ & 1.0198 & 1.0284 & 1.0120 & 1.0600 & 1.0600 & 1.0600 & 1.0600 & 1.0600 & 1.0528 & 1.0600 \\
\hline & $Q$ (MVAR) & 33.42 & 39.05 & 65.06 & 291.38 & 303.03 & 285.36 & 199.18 & 171.50 & 236.18 & 185.40 \\
\hline \multirow[t]{2}{*}{ Decentralized LR } & $V(\mathrm{pu})$ & 1.0600 & 1.0600 & 1.0600 & 1.0365 & 1.0359 & 1.0468 & 1.0600 & 1.0600 & 1.0500 & 1.0600 \\
\hline & $Q$ (MVAR) & 131.32 & 63.05 & 92.74 & 243.63 & 245.52 & 223.01 & 187.53 & 164.13 & 214.23 & 182.62 \\
\hline \multirow[t]{2}{*}{ Centralized LI } & $V(\mathrm{pu})$ & 1.0579 & 1.0600 & 1.0600 & 1.0600 & 1.0524 & 1.0547 & 1.0600 & 1.0600 & 1.0565 & 1.0600 \\
\hline & $Q$ (MVAR) & 103.61 & 53.69 & 86.08 & 279.59 & 255.82 & 217.12 & 173.79 & 157.91 & 224.22 & 166.09 \\
\hline \multirow[t]{2}{*}{ Decentralized ENM LI } & $V(\mathrm{pu})$ & 1.0188 & 1.0280 & 1.0168 & 1.0600 & 1.0507 & 1.0600 & 1.0600 & 1.0600 & 1.0544 & 1.0600 \\
\hline & $Q$ (MVAR) & 31.31 & 38.54 & 71.23 & 302.75 & 275.91 & 290.64 & 200.14 & 171.93 & 243.58 & 183.90 \\
\hline
\end{tabular}

Table 4

The value of control variables for New England 39-bus system in low loading pattern when areas apply the same objective function.

\begin{tabular}{|c|c|c|c|c|c|c|c|c|c|c|c|}
\hline & & \multicolumn{3}{|l|}{ Area A } & \multicolumn{3}{|l|}{ Area B } & \multicolumn{4}{|l|}{ Area C } \\
\hline & & $\mathrm{G}_{1}$ & $\mathrm{G}_{8}$ & $\mathrm{G}_{9}$ & $\mathrm{G}_{2}$ & $\mathrm{G}_{3}$ & $\mathrm{G}_{10}$ & $\mathrm{G}_{4}$ & $\mathrm{G}_{5}$ & $\mathrm{G}_{6}$ & $\mathrm{G}_{7}$ \\
\hline \multirow[t]{2}{*}{ Non-coordinated } & $V(\mathrm{pu})$ & 0.9912 & 0.9935 & 0.9561 & 1.0600 & 1.0600 & 1.0493 & 1.0600 & 1.0600 & 1.0534 & 1.0600 \\
\hline & $Q$ (MVAR) & -25.66 & -17.74 & -17.52 & 250.54 & 262.49 & 226.04 & 170.17 & 144.32 & 194.97 & 151.76 \\
\hline \multirow[t]{2}{*}{ Centralized } & $V(\mathrm{pu})$ & 1.0600 & 1.0600 & 1.0600 & 1.0270 & 1.0271 & 1.0440 & 1.0600 & 1.0600 & 1.0498 & 1.0600 \\
\hline & $Q$ (MVAR) & 97.89 & 34.87 & 38.56 & 162.97 & 164.56 & 149.95 & 140.57 & 129.79 & 150.19 & 138.77 \\
\hline \multirow[t]{2}{*}{ Centralized NC } & $V(\mathrm{pu})$ & 1.0600 & 1.0600 & 1.0600 & 0.9996 & 1.0004 & 1.0185 & 1.0434 & 1.0463 & 1.0309 & 1.0439 \\
\hline & $Q$ (MVAR) & 146.92 & 64.26 & 58.24 & 151.75 & 154.30 & 130.82 & 138.81 & 139.43 & 146.37 & 146.06 \\
\hline \multirow[t]{2}{*}{ Decentralized ENM } & $V(\mathrm{pu})$ & 0.9777 & 0.9738 & 0.9400 & 1.0600 & 1.0600 & 1.0600 & 1.0600 & 1.0600 & 1.0518 & 1.0600 \\
\hline & $Q$ (MVAR) & -44.37 & -46.37 & -13.04 & 253.08 & 267.99 & 275.28 & 179.37 & 148.50 & 198.88 & 159.22 \\
\hline \multirow[t]{2}{*}{ Decentralized LR } & $V(\mathrm{pu})$ & 1.0600 & 1.0600 & 1.0600 & 1.0270 & 1.0271 & 1.0440 & 1.0600 & 1.0600 & 1.0498 & 1.0600 \\
\hline & $Q$ (MVAR) & 97.89 & 34.87 & 38.56 & 162.97 & 164.56 & 149.95 & 140.57 & 129.79 & 150.19 & 138.77 \\
\hline \multirow[t]{2}{*}{ Centralized LI } & $V(\mathrm{pu})$ & 1.0514 & 1.0600 & 1.0600 & 1.0539 & 1.0481 & 1.0523 & 1.0600 & 1.0600 & 1.0553 & 1.0600 \\
\hline & $Q$ (MVAR) & 45.72 & 32.75 & 33.84 & 204.17 & 186.10 & 145.91 & 127.50 & 123.89 & 156.74 & 124.18 \\
\hline \multirow[t]{2}{*}{ Decentralized ENM LI } & $V(\mathrm{pu})$ & 0.9814 & 0.9789 & 0.9400 & 1.0600 & 1.0428 & 1.0600 & 1.0600 & 1.0600 & 1.0544 & 1.0600 \\
\hline & $Q$ (MVAR) & -34.78 & -36.00 & -14.89 & 272.06 & 216.44 & 279.33 & 180.70 & 149.10 & 210.41 & 156.78 \\
\hline
\end{tabular}

- Same objective functions.

- Different objective functions.

- Non-collaborative strategies.

In the non-collaborative strategy, one area can provide biased information on the constraints/objectives to increase its own benefit. All the optimizations contain nonlinear equality and inequality constraints. The non-linear optimization problems are solved using "fmincon" with interior-point algorithm in MATLAB R2011a.

\subsection{Same objective functions}

In this subsection TSOs apply the same objective function given by (5) where $\left(\omega_{1}=1\right)$ and $\left(\omega_{2}, \omega_{3}=0\right)$. The generators voltage set point and their reactive power output for the high and low loading patterns are reported in Tables 3 and 4, respectively. The total cost (objective function value) for the high and low loading patterns are presented in Figs. 6 and 7, respectively. The horizontal axis demonstrates the numbers of iterations since the decentralized approaches are iterative.

For both loading patterns, Centralized has the lowest cost and the distance of the other approaches to this solution is considered as sub-optimality. Decentralized LR converges to Centralized with negligible sub-optimality. Although Non-Coordinated solutions don't have infeasibility, there is high sub-optimality. Centralized

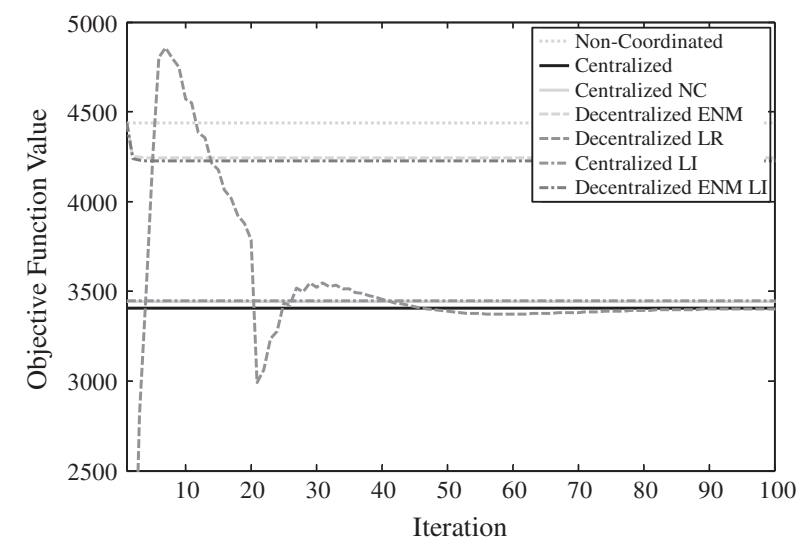

Fig. 6. MAVR for New England 39-bus system in high loading pattern when areas apply the same objective function.

NC provides economically fair solution by accepting a small suboptimality. Decentralized ENM converges to an operating point that does not have necessarily lower cost than Non-coordinated. For instance, the cost of Decentralized ENM is higher than 


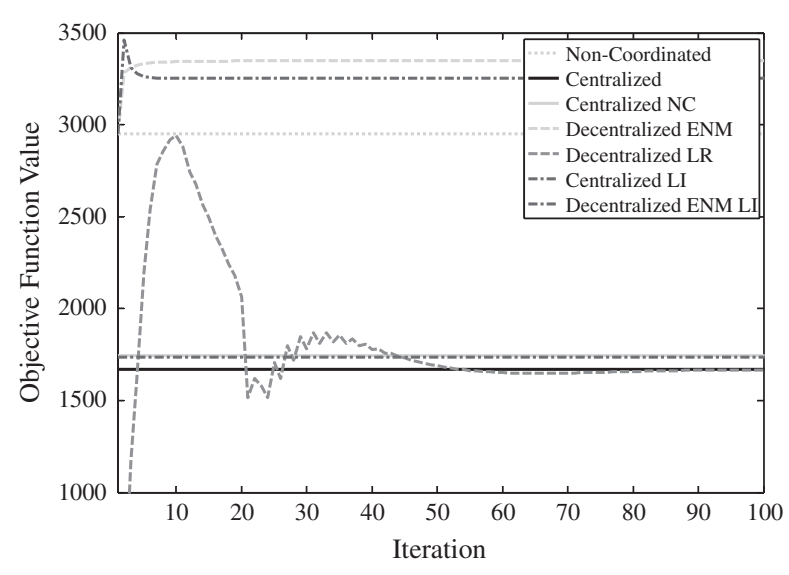

Fig. 7. MAVR for New England 39-bus system in low loading pattern when areas apply the same objective function.

Non-coordinated in low loading pattern while it is the contrary in high loading pattern. As a result, lower sub-optimality is not guaranteed by Decentralized ENM. However, Decentralized ENM LI effectively reduce the sub-optimality of Decentralized ENM and the number of iterations as well. The total cost of Centralized LI increases in comparison with Centralized. However, this solution is close to the solution of Centralized NC. The higher is the $\omega_{4}$, the higher is the total cost because each area should provide its own reactive power resource.

\subsection{Different objective functions}

In this subsection each area applies different objective function. It is assumed that $\mathrm{TSO}_{\mathrm{A}}$ applies minimization of QSQ $\left(\omega_{1}=1\right.$, $\left.\omega_{2}, \omega_{3}=0\right)$. $\mathrm{TSO}_{\mathrm{B}}$ minimization of active power losses $\left(\omega_{2}=1\right.$, $\left.\omega_{1}, \omega_{3}=0\right)$, and $\mathrm{TSO}_{\mathrm{C}}$ minimization of the voltage deviation $\left(\omega_{3}=1, \omega_{1}, \omega_{2}=0\right)$. In other words the cost is defined in different ways for every TSO. The total cost is the sum of the cost of all areas. The generators voltage set point and their reactive power output for the high and low loading patterns are given in Tables 5 and 6, respectively. The simulation results for the high and low loading patterns are presented in Figs. 8 and 9, respectively.

In comparison with the case when TSOs apply the same objective function:

- The difference between Non-coordinated and Centralized solutions is relatively higher due to the disparate nature of the TSOs objectives.

- The sub-optimality of Centralized NC increases in comparison with other approaches since in the proposed fair solution, the normalized objective functions impose higher costs to certain areas.

Table 5

The value of control variables for New England 39-bus system in high loading pattern when areas apply different objective functions.

\begin{tabular}{|c|c|c|c|c|c|c|c|c|c|c|c|}
\hline & & \multicolumn{3}{|l|}{ Area A } & \multicolumn{3}{|l|}{ Area B } & \multicolumn{4}{|l|}{ Area C } \\
\hline & & $\mathrm{G}_{1}$ & $\mathrm{G}_{8}$ & $\mathrm{G}_{9}$ & $\mathrm{G}_{2}$ & $\mathrm{G}_{3}$ & $\mathrm{G}_{10}$ & $\mathrm{G}_{4}$ & $\mathrm{G}_{5}$ & $\mathrm{G}_{6}$ & $\mathrm{G}_{7}$ \\
\hline \multirow[t]{2}{*}{ Non-coordinated } & $V(\mathrm{pu})$ & 1.0155 & 1.0197 & 0.9854 & 1.0600 & 1.0600 & 1.0121 & 1.0600 & 1.0600 & 1.0600 & 1.0600 \\
\hline & $Q$ (MVAR) & 76.02 & 53.18 & 51.80 & 329.53 & 335.39 & 181.63 & 208.30 & 173.54 & 280.41 & 183.84 \\
\hline \multirow[t]{2}{*}{ Centralized } & $V(\mathrm{pu})$ & 0.9889 & 0.9888 & 0.9400 & 1.0600 & 1.0600 & 1.0600 & 1.0600 & 1.0600 & 1.0600 & 1.0600 \\
\hline & $Q$ (MVAR) & 7.34 & 9.64 & 30.98 & 313.37 & 328.53 & 331.73 & 220.13 & 178.89 & 288.42 & 189.83 \\
\hline \multirow[t]{2}{*}{ Centralized NC } & $V(\mathrm{pu})$ & 0.9786 & 0.9867 & 0.9956 & 1.0237 & 1.0363 & 1.0493 & 1.0296 & 1.0179 & 1.0325 & 1.0316 \\
\hline & $Q$ (MVAR) & 7.52 & 22.11 & 109.03 & 273.15 & 339.49 & 367.37 & 229.66 & 147.61 & 291.64 & 192.55 \\
\hline \multirow[t]{2}{*}{ Decentralized ENM } & $V(\mathrm{pu})$ & 1.0493 & 1.0600 & 1.0600 & 1.0497 & 1.0519 & 1.0009 & 1.0600 & 1.0600 & 1.0600 & 1.0600 \\
\hline & $Q$ (MVAR) & 122.04 & 89.51 & 98.65 & 293.08 & 300.89 & 113.20 & 185.17 & 163.06 & 255.00 & 169.81 \\
\hline \multirow[t]{2}{*}{ Decentralized LR } & $V(\mathrm{pu})$ & 0.9889 & 0.9888 & 0.9400 & 1.0600 & 1.0600 & 1.0600 & 1.0600 & 1.0600 & 1.0600 & 1.0600 \\
\hline & $Q$ (MVAR) & 7.34 & 9.64 & 30.98 & 313.39 & 328.54 & 331.77 & 220.13 & 178.89 & 288.42 & 189.83 \\
\hline \multirow[t]{2}{*}{ Centralized LI } & $V(\mathrm{pu})$ & 0.9956 & 1.0033 & 0.9828 & 1.0600 & 1.0119 & 1.0359 & 1.0600 & 1.0600 & 1.0600 & 0.9903 \\
\hline & $Q$ (MVAR) & 36.55 & 43.10 & 73.83 & 391.17 & 210.15 & 286.94 & 247.66 & 191.33 & 407.04 & 33.63 \\
\hline \multirow[t]{2}{*}{ Decentralized ENM LI } & $V(\mathrm{pu})$ & 1.0451 & 1.0600 & 1.0600 & 1.0600 & 1.0064 & 1.0600 & 1.0600 & 1.0600 & 1.0600 & 1.0600 \\
\hline & $Q$ (MVAR) & 72.92 & 77.18 & 96.47 & 339.62 & 135.08 & 272.67 & 188.10 & 164.39 & 258.23 & 171.59 \\
\hline
\end{tabular}

Table 6

The value of control variables for New England 39-bus system in low loading pattern when areas apply different objective functions.

\begin{tabular}{|c|c|c|c|c|c|c|c|c|c|c|c|}
\hline & & \multicolumn{3}{|l|}{ Area A } & \multicolumn{3}{|l|}{ Area B } & \multicolumn{4}{|l|}{ Area C } \\
\hline & & $\mathrm{G}_{1}$ & $\mathrm{G}_{8}$ & $\mathrm{G}_{9}$ & $\mathrm{G}_{2}$ & $\mathrm{G}_{3}$ & $\mathrm{G}_{10}$ & $\mathrm{G}_{4}$ & $\mathrm{G}_{5}$ & $\mathrm{G}_{6}$ & $\mathrm{G}_{7}$ \\
\hline \multirow[t]{2}{*}{ Non-coordinated } & $V(\mathrm{pu})$ & 0.9912 & 0.9935 & 0.9561 & 1.0512 & 1.0543 & 1.0044 & 1.0600 & 1.0600 & 1.0600 & 1.0600 \\
\hline & $Q$ (MVAR) & 10.25 & 1.38 & -11.02 & 262.71 & 279.90 & 128.28 & 175.20 & 145.43 & 229.92 & 147.65 \\
\hline \multirow[t]{2}{*}{ Centralized } & $V(\mathrm{pu})$ & 0.9938 & 0.9981 & 0.9739 & 1.0381 & 1.0394 & 1.0189 & 1.0599 & 1.0599 & 1.0599 & 1.0599 \\
\hline & $Q$ (MVAR) & 0.07 & 0.07 & 0.10 & 230.70 & 240.65 & 166.50 & 173.92 & 144.61 & 225.47 & 146.00 \\
\hline \multirow[t]{2}{*}{ Centralized NC } & $V(\mathrm{pu})$ & 0.9989 & 1.0069 & 0.9695 & 1.0086 & 1.0084 & 1.0291 & 1.0125 & 1.0155 & 1.0144 & 1.0173 \\
\hline & $Q($ MVAR) & 51.02 & 47.33 & 20.28 & 210.94 & 222.94 & 245.84 & 142.31 & 142.78 & 202.38 & 146.45 \\
\hline \multirow[t]{2}{*}{ Decentralized ENM } & $V(\mathrm{pu})$ & 0.9738 & 0.9747 & 0.9400 & 1.0503 & 1.0534 & 1.0094 & 1.0600 & 1.0600 & 1.0600 & 1.0600 \\
\hline & $Q$ (MVAR) & -17.87 & -18.47 & -5.15 & 268.20 & 287.86 & 164.50 & 185.40 & 150.05 & 241.11 & 153.80 \\
\hline \multirow[t]{2}{*}{ Decentralized LR } & $V(\mathrm{pu})$ & 0.9939 & 0.9981 & 0.9741 & 1.0380 & 1.0394 & 1.0189 & 1.0600 & 1.0600 & 1.0600 & 1.0600 \\
\hline & $Q$ (MVAR) & 0.07 & 0.07 & 0.10 & 230.40 & 240.33 & 166.03 & 173.77 & 144.80 & 225.37 & 146.10 \\
\hline \multirow[t]{2}{*}{ Centralized LI } & $V(\mathrm{pu})$ & 0.9693 & 0.9752 & 0.9552 & 1.0600 & 0.9751 & 1.0030 & 1.0600 & 1.0600 & 0.9849 & 0.9400 \\
\hline & $Q$ (MVAR) & 16.81 & 20.09 & 34.82 & 426.94 & 108.26 & 201.91 & 284.47 & 194.81 & 206.38 & 8.26 \\
\hline \multirow[t]{2}{*}{ Decentralized ENM LI } & $V(\mathrm{pu})$ & 1.0059 & 1.0108 & 0.9899 & 1.0600 & 0.9727 & 1.0600 & 1.0600 & 1.0600 & 1.0600 & 1.0600 \\
\hline & $Q$ (MVAR) & 2.33 & 3.00 & 6.04 & 339.88 & 12.94 & 275.24 & 174.70 & 145.21 & 229.39 & 147.35 \\
\hline
\end{tabular}




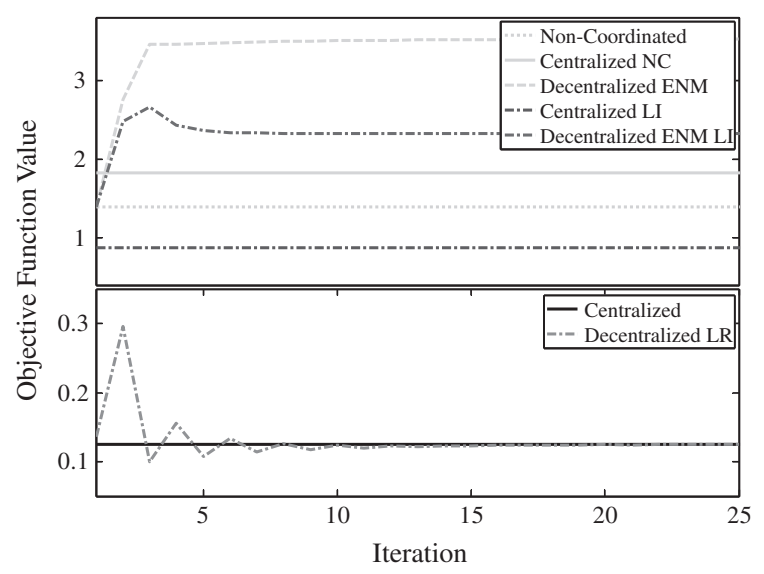

Fig. 8. MAVR for New England 39-bus system in high loading pattern when areas apply different objective functions.

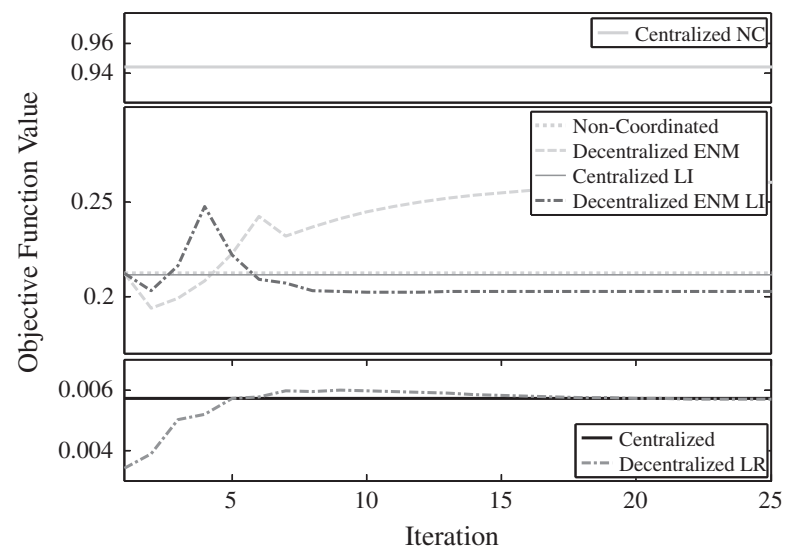

Fig. 9. MAVR for New England 39-bus system in low loading pattern when areas apply different objective functions.

Similar to the case with the same objective functions:

- Decentralized ENM has significant sub-optimality.

- Decentralized LR converges to Centralized with negligible suboptimality. The convergence is clearly shown in the bottom of Figs. 8 and 9.

- Centralized LI gives a solution that is not far-off the solution of Centralized NC.

- Decentralized ENM LI reduces the sub-optimality and the required number of iterations for Decentralized ENM.

\subsection{Non-collaborative strategy}

In these simulations all TSOs apply the same objective function given by (5) where $\left(\omega_{1}=1\right)$ and $\left(\omega_{2}, \omega_{3}=0\right)$. It is assumed that $\mathrm{TSO}_{\mathrm{B}}$ aims to provide incorrect information of its own network for other TSOs. These strategic behaviors could be applied on various information e.g. the generator limits, voltage limits and objective functions. The solution of every approach for different non-collaborative strategies is compared with the result of Centralized with the same objective function (Centralized Ref.) given in 4-1. An optimization approach is called robust against a strategic behavior if the results of non-collaborative and collaborative strategies become similar. Here, the simulation results are not presented due to page number limit. Only the conclusions are discussed. It is interesting to mention that for all strategies and approaches, the sub-optimality in high loading pattern is rather lower than in the case of low loading pattern, since in the former, the system is closer to its limits and the feasible region of the solution is more limited.

\subsubsection{Generator limits}

In this strategy, $\mathrm{TSO}_{\mathrm{B}}$ applies fifty percent lower limit for the maximum generators reactive power. Therefore, reactive power output of area $\mathrm{B}$ is limited and the costs of its neighbors increase. In the high loading pattern, there is no solution to non-coordinated approach due to infeasibility in the optimization of area $B$, while a solution exist for low loading pattern. Centralized and Decentralized LR approaches approximately have the same sub-optimality regarding to Centralized Ref. case. This sub-optimality is close to zero at low loading pattern while it increases at high loading pattern. The reason is that at low loading pattern the generators maximum reactive power constraints are not reached but at the high loading pattern these constraints are active. Centralized NC is not robust against this strategy and the total cost goes higher. For Decentralized ENMs and Centralized LI the total cost increases (decreases) at low (high) loading pattern. Note that the decentralized approaches here, including Decentralized ENMs and Decentralized LR, require fictitious reactive power sources in infeasible iterations, as the special treatment mentioned in $2-2-3$, to avoid the infeasibility.

\subsubsection{Voltage limits}

For this strategy, $\mathrm{TSO}_{\mathrm{B}}$ applies the voltage deviation within ten percent instead of six percent (disclose). The centralized approach is robust against this strategy behavior while Decentralized LR has sub-optimality. The result of Centralized NC in high (low) loading pattern has higher (lower) cost. The sub-optimality increases for the other approaches. Furthermore, $\mathrm{TSO}_{\mathrm{B}}$ may apply the voltage deviation within six percent but inform the other areas that ten percent voltage deviation is acceptable for this area (non-disclose). Centralized and Centralized LI approaches are immune against this strategy. The total cost of Centralized NC in high (low) loading increase (decrease). For the other approaches the sub-optimality decreases. Generally, these strategies do not improve the objective of area $B$ necessarily, and even they may effect adversely on its objective [13].

\subsubsection{Objective functions}

It is assumed that $\mathrm{TSO}_{\mathrm{B}}$ declares its cost function as $2 \times \mathrm{OF}$ and $O F^{2}$, respectively. The first strategy is linear transformation of the objective function. The results of Non-Coordinated, Centralized NC and Decentralized ENM are robust against this strategy. In both loading patterns, the sub-optimality of Centralized LI decrease. In contrast, the sub-optimality increases for Centralized, Decentralized LR and Decentralized ENM LI. In the second strategy $\mathrm{TSO}_{\mathrm{B}}$ minimizes the square of its cost. Simulation results demonstrate that Non-Coordinated, Centralized NC and Decentralized ENM are robust against this strategy. The sub-optimality increases for the other approaches. The robustness of the different approaches to the non-collaborative strategies is summarized in Table 7.

Table 7

The robustness of different approaches to the strategic behavior of $\mathrm{TSO}_{\mathrm{B}}$.

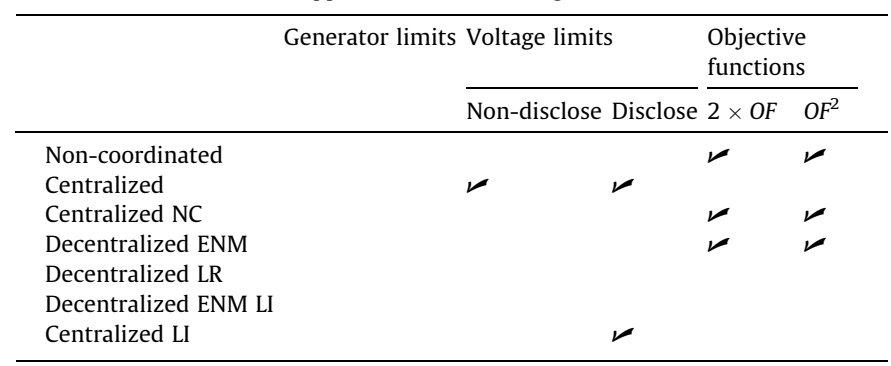




\section{Conclusion}

This paper investigated the inter-area optimal voltage and reactive power control. The state of the art in practice and research are studied and the necessity of MAVR is enlightened. Several centralized and decentralized approaches are examined to investigate their effectiveness for the voltage and reactive power control in MAPS. The proposed formulation benefits from the distributed slack bus model for the response of generators to the active power losses. This modeling allows an easier implementation of the optimization approaches and also improves the optimization results.

In collaborative environment the solution of Centralized approach is considered as the reference case to obtain sub-optimality of other approaches. Decentralized LR practically always converges to zero sub-optimality but it requires more iteration and more information exchange between neighboring areas. Decentralized ENM converges with less number of iterations but with high suboptimality. Additional limits on the voltage difference at both ends of interconnections effectively reduce the sub-optimality of Decentralized ENM while the number of iterations decreases a little. Non-coordinated operation always has high sub-optimality and non-feasibility is also possible. Centralized NC proposes an economically fair solution with low sub-optimality for the same objective functions and higher sub-optimality when the objective functions are different. The solution of Centralized with limits on the reactive power flow at interconnections is close to the solution of Centralized NC.

The discussed approaches are not robust against all kinds of strategic behaviors. Therefore, a collaborative framework should be realized for an appropriate MAVR. The non-collaborative behaviors can be avoided for instance by improving the power system monitoring. However, additional limits on the difference of the voltage of both ends of the interconnections and reactive power flow limits in the interconnections are effective against strategic behaviors when limiting the strategic decisions of one TSO within its own area.

\section{Acknowledgement}

The authors thankfully acknowledge Swiss Electric Research as the results reported in this paper have been carried out within the framework of the research project "Security of Multi-Area Power Systems (MARS)".

\section{References}

[1] Federal Energy Regulatory Commission Staff Report. Principles for efficient and reliable reactive power supply and consumption. Docket No. AD05-1-000; February 2005.

[2] Lu W, Bésanger Y, Zamaï E, Radu D. Blackouts: description, analysis and classification. In: 6th WSEAS int conf power syst, Lisbon; 2006.

[3] Alizadeh Mousavi O, Cherkaoui R. Literature survey on fundamental issues of voltage and reactive power control. <http://mars.ethz.ch/en/researh-andpublications/publications.html> [October 2011].
4] Zhang A, Li H, Liu F, Yang $\mathrm{H}$. A coordinated voltage/reactive power control method for multi-TSO power systems. Int J Electr Power Energy Syst 2012;43:20-8.

[5] Gomez-Exposito A, Conejo AJ, Canizares C. Electric energy systems analysis and operation. Taylor \& Francis; 2009.

[6] ENTSO-E operation hand book. <https://www.entsoe.eu> [March 2009].

[7] Van Cutsem T, Vournas C. Voltage stability of electric power systems. Kluwer Academic Publisher; 1998.

[8] Vu H, Pruvot P, Launay C, Harmand Y. An improved voltage control large-scale power system. IEEE Trans Power Syst 1996;11(3):1295-303.

[9] Corsi S. Wide area voltage regulation \& protection. In: IEEE Bucharest PowerTech, Bucharest; 2009.

[10] Phulpin Y, Begovic M, Ernst D. Coordination of voltage control in a power system operated by multiple transmission utilities. In: REP symposium, Buzios; 2010

[11] Phulpin Y. Coordination of reactive power scheduling in a multi-area power system operated by independent utilities. PhD thesis in Georgia Institute of Technology; 2009.

[12] Arnold M, Knöpfli S. Multi-area control in electric power systems. Master's thesis, ETH Zürich; 2006.

[13] Phulpin Y, Begovic M, Petit M, Ernst D. A fair method for centralized optimization of multi TSO power system. Int J Electr Power Energy Syst 2009;31:482-8.

[14] Phulpin Y, Begovic M, Petit M, Ernst D. On the fairness of centralized decision making strategies in multi TSO power systems. In: Power syst comput conf, Glasgow; 2008.

[15] Phulpin Y, Begovic M, Petit M. External netwrok modeling for MVAr scheduling in multi area power systems. In: PowerTech, Lausanne; 2007.

[16] Phulpin Y, Begovic M, Petit M, Heyberger JB, Ernst D. Evaluation of network equivalents for voltage optimization in multi-area power systems. IEEE Trans Power Syst 2009;24(2):729-43.

[17] Phulpin Y, Begovic M, Petit M, Ernst D. Decentralized reactive power dispatch for a time varying multi TSO system. In: Hawaii int conf syst sci; 2009.

[18] Expósito AG, Ramos JLM, Santos JR. Slack bus selection to minimize the system power imbalance in load-flow studies. IEEE Trans Power Syst 2004;19(2):987-95.

[19] Natarajan R. Computer-aided power system analysis. New York, Basel: Marcel Dekker Inc.; 2002.

[20] Alizadeh Mousavi O, Bozorg M, Cherkaoui R. Preventive reactive power management for improving voltage stability margin. Electr Power Syst Res 2013;96:36-46.

[21] Rosehart W, Canizares C, Quintana V. Multiobjective optimal power flows to evaluate voltage security costs in power networks. IEEE Trans Power Syst 2003;18(2):578-87.

[22] Tamimi B, Canizares C, Vaez-Zadeh S. Effect of reactive power limit modeling on maximum system loading and active and reactive power markets. IEEE Trans Power Syst 2010;25(2):1106-16.

[23] Hug-Glanzmann G, Andersson G. Decentralized optimal power flow control for overlapping areas in power systems. IEEE Trans Power Syst 2009;24:327-36.

[24] Phulpin Y, Begovic M, Petit M. Impact of non-Coordinated MVAr scheduling strategies in multi area power systems. In: IEEE power engineering society general meeting, Tampa, Florida; 2007.

[25] Zima M, Ernst D. On multi-area control in electric power systems. In: Proceedings of the 15th power systems computation conference, Liege; 2005.

[26] Arnold M, Knopfli S, Andersson G. Improvement of OPF decomposition methods applied to multi-area power systems. In: IEEE Power Tech, Lausanne; 2007.

[27] TSO Security Cooperation, TSC; 2011. <http://www.tso-securitycooperation.eu/>.

[28] Granada M, Rider MJ, Mantovani J, Shahidehpour M. A decentralized approach for optimal reactive power dispatch using a Lagrangian decomposition method. Electr Power Syst Res 2012;89:148-56.

[29] Yan W, Wen L, Li W, Chung CY, Wong KP. Decomposition-coordination interior point method and its application to multi-area optimal reactive power flow. Int J Electr Power Energy Syst 2011;33:55-60.

[30] Biskas PN, Bakirtzis A. Decentralised OPF of large multiarea power systems. IEE Proc Gener Trans Distrib 2006;153:99-105.

[31] Marinakis A, Glavic M, Van Cutsem T. Control of phase shifting transformers by multiple transmission system operators. In: PowerTech, Lausanne; 2007. 